\title{
Ambientes primarios de desarrollo y habilidades sociocognitivas en escolares con comportamientos disruptivos
}

Socorro Francinet Hernández Herrera ${ }^{1}$

Rolando Alfredo Ardón Ledezma²

\section{RESUMEN}

El presente estudio es tipo descriptivo mixto, predominantemente cualitativo. El principal objetivo fue conocer el funcionamiento de los ambientes primarios de desarrollo y las habilidades sociocognitivas de escolares con comportamientos disruptivos de la Escuela John F. Kennedy de Tegucigalpa, Honduras. Se evaluaron 15 participantes (14 varones, 1 mujer) entre las edades de 6 a 10 años, utilizando técnicas observacionales, psicométricas, proyectivas y entrevistas.

Los resultados muestran que en el ambiente familiar predomina el tipo de familia biparental y en menor medida se presenta la estructura monoparental. Sobre las relaciones con el padre y la madre, todos los participantes mostraron conflictos que se caracterizan por relaciones hostiles, carentes de afecto y comunicación. En las relaciones fraternas se mostraron conflictos de rivalidad, hostilidad y distancia emocional. El estilo de crianza en la mayoría de los participantes fue el autoritario y solo en uno se presentó el estilo permisivo. Las prácticas disciplinarias en todos los casos son los castigos físicos y restrictivos.

El ambiente escolar posee condiciones físicas idóneas. En las interacciones entre compañeros se identificaron dificultades de integración en el grupo debido al rechazo por sus conductas. Todos los participantes mostraron conflictos por agresividad y molestias con sus compañeros. Los profesores muestran un estilo disciplinario ambivalente ante las disrupciones.

Con relación a las habilidades sociocognitivas enfocadas a solución de problemas en

\footnotetext{
${ }^{1}$ Beneficiaria de una beca de pregrado de la DICYP, estudiante de la Carrera de Psicología, Facultad de Ciencias Sociales, UNAH: francih220@hotmail.com

${ }^{2}$ Asesor, Carrera de Psicología, Facultad de Ciencias Sociales, UNAH: rolandoledezma@gmail.com.
} 
las relaciones interpersonales, la mayoría presenta una adecuada habilidad, por lo que se puede decir que los participantes poseen las herramientas mentales para actuar adecuadamente en situaciones problemáticas de interacción social.

Palabras clave: perspectiva bioecológica, microsistemas, estilos de crianza, relaciones entre pares, teoría sociocultural, habilidades sociocognitivas.

\section{ABSTRACT}

The study has a mixed descriptive methodological approach, with qualitative predominance. The main objective was to understand the functioning of the primary development environment and socio-cognitive skills of school children with disruptive behavior in the John F. Kennedy School of Tegucigalpa, Honduras. 15 participants (14 males, 1 female) between the ages of 6 to 10 years were evaluated, using observational, psychometric, projective and interview techniques.

The results show that a two-parent family prevails over a single-parent structure in regards to family environment. Related to the father and mother, all participants showed conflicts characterized by hostile relations, lacking affection and communication. In fraternal relations, rivalry conflicts, hostility and emotional distance were found. Most parenting styles were described as authoritarian and just in one case the permissive style was described. The disciplinary practices, in all cases are the physical and restrictive punishments.

The school environment has ideal physical conditions. In group interactions, integration difficulties were identified, due to rejection by their conduct. All participants showed aggressiveness conflicts with peers. Teachers showed an ambivalent disciplinary style toward disruptive conduct.

Regarding the socio-cognitive skills focused on solving problems in interpersonal relationships, most students have adequate skills, so we can say that the participants have the mental tools to act appropriately in problematic situations of social interaction.

Keywords: bioecologic perspective, microsistems, parenting style, peer relationships, sociocultural theory, social and cognitive skills. 


\section{INTRODUCCIÓN}

Uno de los problemas que se presentan en los centros educativos es lidiar con actos de indisciplina, violencia o desafíos a las normas de convivencia dentro del aula, los cuales se denominan conductas disruptivas. Estas generan un clima tenso en donde se crean malas relaciones interpersonales, tanto entre profesores y alumnos, como entre los propios alumnos (Moreno y Torrejo, 2003; Fernández, 2006; Calvo, 2011).

La Escuela John F. Kennedy de Tegucigalpa carece de programas de intervención psicopedagógica, por lo que el alumnado con dificultades emocionales y problemas de conducta no recibe ningún tipo de asistencia psicológica que contribuya a mejorar estos problemas.

De acuerdo con Frías, López y Díaz (2003), para intervenir en este problema es necesario analizar todos los factores contextuales en los que se encuentra inmersa la persona, por lo que antes de realizar cualquier actividad encaminada a mejorar el bienestar emocional y el comportamiento de los alumnos, es importante establecer un diagnóstico de la situación de cada uno y analizar las condiciones ambientales, afectivas y cognitivas que están asociadas a sus conductas. Por ello se ha considerado de gran utilidad realizar el presente estudio, pues la información que llegue a obtenerse servirá a las autoridades, al personal docente de la escuela y a los padres de familia para que conozcan cómo es la dinámica psicológica de cada uno de los alumnos que presentan conductas disruptivas.

Bajo la luz de la teoría bioecológica y la sociocultural se describen los ambientes primarios de desarrollo de los escolares con comportamientos disruptivos de la Escuela John F. Kennedy. Se analizan los ambientes familiar y escolar, así como las habilidades cognitivas de los niños para resolver problemas interpersonales. A continuación se presenta la fundamentación teórica del estudio.

\section{Perspectiva bioecológica del desarrollo psicosocial}

La psicología evolutiva explica el desarrollo social y afectivo del ser humano desde diferentes perspectivas teóricas. Para los propósitos del presente estudio se han tomado en cuenta los fundamentos de la teoría bioecológica de Uri Bronfenbrenner (1987). Este modelo propone un aspecto biológico en el cual se reconoce que las personas incorporan su yo biológico al proceso de desarrollo y también una parte ecológica que considera los contextos sociales de desarrollo como ecosistemas, ya 
que interactúan constantemente e influyen unos en otros (Woolfolk, 2010).

De acuerdo con este postulado, si un determinado niño o niña actúa o piensa de cierta manera, se debe a sus propias características personales y la interacción de estas con las características de cada uno de los escenarios en que el niño vive, tanto de forma directa como de forma indirecta (Espinoza, Ochaita y Ortega, 2003). Al hablar de relaciones directas debe entenderse como aquellos contactos que el niño tiene con ambientes cercanos como la familia o sus amistades, mientras que las relaciones indirectas se refieren a contextos más globales que forman parte de su realidad social, como los sistemas político, económico, educativo y religioso.

En cuanto a dichas interacciones, Bronfenbrenner (1987) afirmaba que: "Lo que cuenta para la conducta y el desarrollo es el ambiente, cómo se le percibe, más que cómo pueda existir en la realidad objetiva". Es decir, que lo que influye en la manera de comportarse de los niños es la interpretación subjetiva que cada uno hace de las circunstancias que se presentan en el ambiente y estas circunstancias adquieren un significado individual que se refleja en sus actuaciones.

Y como ya se ha mencionado, las interacciones se desarrollan en diferentes niveles, ubicándose en el primero el microsistema, en él se encuentran las actividades, roles y relaciones íntimas e inmediatas de la persona (Díaz, Frías y López, 2003; Woolfolk, 2010), entre ellas: la familia, amigos, actividades escolares y profesores. El siguiente es el mesosistema, estas son las interacciones entre todos los elementos del microsistema, que a su vez está incluido en un exosistema, el cual se refiere a ambientes sociales que afectan al niño, aun cuando este no sea un participante activo; incluye los recursos de la comunidad, el centro de trabajo de los padres, etc. Todos forman parte del macrosistema, es decir, la sociedad en general con sus leyes, costumbres y valores (Woolfolk, 2010).

\section{Microsistema: ambientes primarios de desarrollo}

En la familia, la escuela y el grupo de pares, se desarrolla una constante dinámica entre actividades, roles y relaciones interpersonales que intervienen en la formación de la personalidad y la conducta (Díaz, Frías y López, 2003). Los diferentes estilos de crianza parental tienen un papel importante en el comportamiento de los niños, por ejemplo, los padres autoritativos aplican un control firme a sus hijos y al mismo tiempo alientan la comunicación y negociación en el establecimiento de las reglas, el resultado de esto es que los niños se vuelven más seguros de sí mismos y muestran mayor autocontrol y competencias sociales. 
Los padres autoritarios adoptan estructuras con reglas rígidas y las imponen a sus hijos, lo que impide que estos puedan intervenir en la toma de decisiones de la familia, esto puede repercutir en conductas retraídas, poco asertivas, rebeldes y agresivas. En cambio, los padres permisivos ejercen poco control sobre sus hijos y suelen ser afectuosos, estos niños también pueden reflejar rebeldía, agresividad, impulsividad e inadaptación social, solo en algunos casos podrían ser dinámicos, extrovertidos y creativos. Los padres indiferentes muestran poco control y afecto a sus hijos y al combinar esto con la hostilidad podrían desarrollarse hasta conductas antisociales (Baucum y Craig, 2009).

En cuanto a la relación entre pares, Clemente, Górriz, Regal y Villanueva (2003), aseguran que la interacción entre iguales, más allá de la familia, escuela y entorno en general, es importante en el contexto de desarrollo para la adquisición de habilidades, actitudes y experiencias necesarias para la socialización, contribuyendo a la adaptación social, emocional y cognitiva de los niños.

Conceptualización de las habilidades sociocognitivas

Ser socialmente habilidoso implica ser capaz de resolver una situación social de forma efectiva, lo cual implica integrar aspectos conductuales, fisiológicos y cognitivos (Luca, Rodríguez y Sureda, 2004). Al respecto, Shure (1996) menciona que: "Las habilidades para organizar las cogniciones y conductas dirigidas hacia metas interpersonales se denominan habilidades sociocognitivas".

\section{Desarrollo de las habilidades sociocognitivas}

La teoría sociocultural de Vygotsky (1978) es esencial para comprender el papel que desempeña el ambiente social en el desarrollo cognoscitivo, pues Vygotsky propone que a través de las interacciones sociales se adquieren herramientas culturales y psicológicas que llegan a internalizarse en cada individuo para constituir las funciones y habilidades cognoscitivas (Woolfolk, 2010).

En sintonía con lo anterior, Ison y Morelato (2008) plantean que todas las personas emplean estrategias y habilidades para enfrentar diversos desafíos y demandas que se presentan en los ambientes interpersonales, lo cual es producto de aprendizajes previamente adquiridos en los diferentes contextos sociales de desarrollo. A su vez, afirman que en dichos contextos pueden adquirirse tanto las conductas socialmente competentes, como también aquellas que son disfuncionales para el niño y de quienes le rodean. 
De acuerdo con estas perspectivas, se pretende describir el funcionamiento de los ambientes primarios de desarrollo, identificando las percepciones y experiencias formadas en estos contextos, así como las fortalezas y las debilidades de los participantes con relación a las habilidades cognitivas para la resolución de problemas interpersonales.

\section{METODOLOGÍA}

\section{Diseño}

Se utiliza el diseño etnográfico de corte transversal (Baptista, Fernández y Hernández, 2007), ya que se estudian las características del grupo de escolares en un determinado tiempo (septiembre-diciembre de 2015).

\section{Participantes}

Los participantes han sido seleccionados a través de un muestreo no probabilístico de casos-tipo, valorando la capacidad operativa de recolección y análisis de datos, así como la naturaleza del problema. La muestra está conformada por 15 alumnos (14 niños y 1 niña, entre 6 y 10 años) con comportamientos disruptivos de la Escuela John F. Kennedy de Tegucigalpa, Honduras.

La elección se basó en la identificación de las características conductuales que Fernández (2006) establece como criterios para la detección del comportamiento disruptivo, las cuales se ubican en 4 categorías: inadecuadas referidas a la tarea, vinculadas con las relaciones entre compañeros, contra las normas del aula e inapropiadas de falta de respeto al profesor.

\section{Intervenciones}

Con la finalidad de profundizar el conocimiento de las características y funcionamiento de los ambientes primarios de desarrollo (familia y escuela) de los niños y niñas con conductas disruptivas, tomando algunos elementos de los modelos de Jerome Sattler (2010), se utilizaron 3 formatos de entrevistas semiestructuradas: padres, maestros y niños. Estas entrevistas se realizaron de forma individualmente a cada una de las personas involucradas en esta fase del proceso de recolección de datos. 
El primer formato se utilizó con 15 familiares responsables de los alumnos seleccionados en la muestra de estudio, el segundo con 10 docentes responsables de las diferentes secciones de primero a quinto grado de la escuela y el tercer formato de entrevista se usó con los 15 estudiantes de la muestra seleccionada.

Se elaboró una guía de registro conductual que pretende describir la ocurrencia de conductas relacionadas con las interacciones en el ambiente escolar. Las categorías de observación que contiene la guía son: comportamientos individuales, relaciones con los pares, relaciones con los profesores, estilo disciplinario del docente y condiciones físicas del aula.

Se utilizó el test de la familia kinética (Burns y Kaufman, 1972), que es una técnica de evaluación psicológica proyectiva gráfica útil para conocer las dificultades de adaptación al medio familiar, los conflictos con figuras parentales y de rivalidad fraterna. Además de los aspectos emocionales, refleja el desarrollo intelectual del niño.

Asimismo, se usó el test de apercepción temática (CAT) (Bellak y Bellak, 1949) que también es una técnica de evaluación psicológica proyectiva. Consiste en 10 láminas que representan figuras de animales en diversas situaciones sociales humanizadas. Evalúa aspectos inconscientes que revelan la dinámica de la personalidad, su manifestación en las relaciones interpersonales y en la interpretación del ambiente, así como motivos y necesidades de logro, poder e intimidad y habilidades de resolución de problemas.

De igual manera, se utilizó el test de evaluación de habilidades cognitivas de solución de problemas interpersonales (EVHACOSPI), (García y Magaz, 1998). En el EVHACOSPI, a través de 6 láminas ilustradas, se plantean distintas interacciones sociales que constituyen un problema para uno de los protagonistas. Las variables que se analizan son: habilidad para identificar situaciones de interacción social que constituyen un problema, habilidad para definir de manera concreta una situación problema, habilidad para generar posibles alternativas que constituyan una solución a un problema interpersonal: amplitud y flexibilidad de pensamiento alternativo, habilidad para anticipar posibles consecuencias asociadas a una determinada forma de resolver un problema interpersonal: amplitud y flexibilidad de pensamiento consecuencial y habilidad para tomar decisiones, eligiendo la mejor alternativa de todas las posibles. 


\section{RESULTADOS}

En los resultados se da a conocer el funcionamiento de los ambientes primarios de desarrollo (se incluyen los ambientes familiar y escolar) y las habilidades sociocognitivas de los alumnos participantes. De estos, se hacen análisis orientados a la comprensión de su influencia en las conductas disruptivas.

\section{Ambiente familiar}

Sobre este se reconocen las características psicosociales que lo integran y se identifica la manera en que los niños y niñas perciben y experimentan las relaciones en este contexto. El tipo de estructura familiar que predominó en los participantes fue el biparental (10), es decir, la familia integrada por ambos cónyuges; solamente cuatro niños provienen de familias monoparentales, en tales casos, la responsabilidad recae en la madre; asimismo, se presentó un caso de familia reconstituida en la cual un cónyuge tiene hijos de uniones anteriores.

Sobre las relaciones con las figuras parentales, o sea, los vínculos establecidos con la madre y el padre, todos los participantes mostraron conflictos que se caracterizan por relaciones hostiles, carentes de afecto y comunicación con ambos padres. Destaca la situación de cuatro participantes que presentan un mayor conflicto con la figura paterna, la que perciben como agresiva y amenazante debido a los castigos físicos que les inflige, lo que les provoca constante temor. Por otra parte, dos de los niños perciben sus relaciones parentales como ambivalentes, entre el afecto y el rechazo.

El estilo de crianza predominante en los participantes fue el autoritario (14), este se caracteriza por estructuras con reglas rígidas e impositivas, lo cual puede repercutir en conductas retraídas, poco asertivas, rebeldes y agresivas. Solo se identificó un participante que vive bajo el estilo de crianza permisivo, en este caso los padres ejercen poco control sobre sus hijos y suelen ser afectuosos, estos niños también pueden reflejar rebeldía, agresividad, impulsividad e inadaptación social (Baucum y Craig, 2009). Todos los participantes reciben un modo de disciplina basado en castigos físicos y restrictivos. En las relaciones fraternas ocho participantes mostraron conflictos de rivalidad debido a que sienten que sus hermanos reciben mayor atención y afecto; dos niños perciben hostilidad en sus relaciones y otros dos se sienten distantes de sus hermanos. Solamente tres niños no mostraron conflictos en sus relaciones fraternas. Debido a la manera en que los niños se relacionan con sus 
familiares, perciben el ambiente como hostil (8), carente de afecto (6) y restrictivo (7).

\section{Ambiente escolar}

Al igual que en el apartado anterior, en este se reconocen las principales características psicosociales que integran el ambiente, pero en este caso el escolar, se identifica la manera en que los niños y niñas experimentan las relaciones en este contexto. Los elementos físicos del aula de clases (que es donde los niños tienen sus interacciones) se caracterizan por una adecuada iluminación, ventilación y espacio; se cuenta con pupitres para cada alumno, por lo que el espacio físico es el idóneo para el proceso de enseñanza-aprendizaje. En las interacciones con sus pares en el ecosistema del aula, once participantes mostraron ser amistosos con sus compañeros y que se integran fácilmente al grupo, pero cuatro tenían dificultades para integrarse debido al rechazo que sus compañeros tenían hacia ellos por las disrupciones que ocasionaban. Se observó que cinco de los participantes eran influenciados por su grupo de amigos para crear disrupciones en el aula. Asimismo, las interacciones en el aula en ocasiones se tornaban en molestias como burlas a compañeros (7) y peleas (7).

Sobre el estilo disciplinario del profesor, se observó a seis de ellos; todos mostraron conductas ambivalentes ante las disrupciones, ya que en ocasiones se mostraban indecisos, poco firmes y condescendientes, lo que provocaba que perdieran el control del grupo. Por otra parte, hubo momentos en los que se mostraban irritados, era común observar que expresaran enojo y que gritaran, castigaban a los niños suspendiéndoles el recreo y una maestra dejó parado a un alumno durante la clase; otra de ellas amenazaba con asignar tarea a los niños si se seguían "portando mal" o con informar a sus padres acerca de su conducta. De igual manera, en una ocasión una maestra amenazó a sus alumnos ofreciendo castigos con la regla. Es evidente que ninguno de los recursos empleados para mantener el orden fue útil para los maestros.

\section{Habilidades sociocognitivas}

Con el propósito de determinar las fortalezas y las debilidades en el desarrollo de las habilidades sociocognitivas de los niños, se encontró que once presentan adecuada y cuatro inadecuada habilidad cognitiva para la solución de problemas en las relaciones interpersonales, por lo que se puede decir que la mayoría posee un pensamiento amplio y flexible. 
De manera detallada, sobre la habilidad para identificar situaciones de interacción social que constituyen un problema, los 15 niños muestran un nivel adecuado. Sobre la habilidad para definir de manera concreta una situación problema, 10 de los participantes mostraron un desempeño adecuado, en cambio 5 participantes indicaron debilidad para realizar este proceso mental. Sobre la habilidad para generar posibles alternativas que constituyan una solución a un problema interpersonal, 8 niños mostraron un desempeño adecuado, lo cual implica una fortaleza en la amplitud y flexibilidad del pensamiento alternativo, el cual requiere buscar diversas soluciones a un mismo problema interpersonal; sin embargo, 7 niños mostraron debilidad para realizar este proceso mental.

Sobre la habilidad para anticipar posibles consecuencias asociadas a una determinada forma de resolver un problema interpersonal, 11 mostraron un desempeño adecuado, lo cual implica una fortaleza en la amplitud y flexibilidad de pensamiento consecuencial, lo que requiere la capacidad para razonar y prever las consecuencias de los propios actos en un problema interpersonal; no obstante, 4 niños mostraron debilidad. Sobre la habilidad para tomar decisiones, eligiendo la mejor alternativa de todas las posibles, 8 niños mostraron un desempeño adecuado y 7 niños mostraron debilidad. Como última parte del proceso, sobre el afrontamiento ante situaciones de interacción social, varía entre conductas reactivas como la agresividad e impulsividad (5) y conductas más inhibidas como la evasión (1), el temor al rechazo (4), sentimientos de fracaso (4) y sumisión (1).

La manera de afrontar problemas de interacción social está ligada al aprendizaje social que se desarrolla en los ambientes primarios. Los estilos de afrontamiento identificados tienen relación especialmente con la percepción y experiencias que los niños han tenido en su ambiente familiar, los cuales evidentemente han producido conflictos emocionales como los ya mencionados.

\section{DISCUSIÓN Y CONCLUSIONES}

En la estructura familiar predomina el tipo biparental y en menor medida se presenta la estructura monoparental. Sobre las relaciones con el padre y la madre, todos los participantes mostraron conflictos que se caracterizan por relaciones hostiles, carentes de afecto y comunicación con ambos padres; en algunos casos se suma la ambivalencia entre el afecto y el rechazo. Al respecto Rodríguez, del Barrio y Carrasco (citados por Malonda, 2012) encontraron que la inconsistencia en el 
comportamiento de ambos padres en las prácticas de crianza tiene relaciones significativas con alteraciones emocionales y conductuales, como mayores índices de la sintomatología depresiva y agresión física y verbal, falta de control y de autoeficacia.

Es probable que los conflictos de rivalidad fraterna sean reforzadores del comportamiento inapropiado, el cual puede estar ligado al deseo de que les presten mayor atención.

Con relación a los estilos de crianza, el estilo autoritario influye en la rebeldía y agresividad que manifiestan los niños con comportamientos disruptivos, al igual que el estilo permisivo. De forma general, se puede observar que en el ambiente familiar destaca la poca funcionalidad afectada por la agresividad, la falta de afecto y problemas en la comunicación; esto influye significativamente en las conductas disruptivas, pues favorece que se replique la violencia y las malas relaciones interpersonales.

En las interacciones entre compañeros se presenta amistad en algunos casos, sin embargo, en otros no hay integración en el grupo debido al rechazo por sus conductas. Todos los participantes tienen conflictos por agresividad y molestias con los compañeros. Los profesores presentan un estilo disciplinario ambivalente ante las disrupciones, pues a veces se comportan indecisos, poco firmes y condescendientes; pero otras veces muestran enojo y aplican castigos. Como interpretación a esta situación, se puede decir que las predisposiciones de agresividad que aprenden los niños en sus hogares vienen a replicarse en la escuela, por lo que se retroalimenta esta conducta con los compañeros y al intervenirse ineficazmente a través de los profesores, con ambivalencia entre permisividad y dominancia, no se logra disminuir la problemática, al contrario, se mantiene.

Con respecto a las habilidades sociocognitivas enfocadas en la solución de problemas en las relaciones interpersonales, la mayoría posee las herramientas mentales para actuar adecuadamente en situaciones problemáticas de interacción social; sin embargo, si presentan problemas es probable que los factores ambientales, principalmente el aprendizaje obtenido en el ambiente familiar y la reproducción de conductas disruptivas entre compañeros, sea el reforzamiento de su desajuste conductual. En el caso de los cuatro que presentan habilidades inadecuadas, puede decirse que este es un factor influyente en la presencia de sus conductas disruptivas, sumado a otros factores ambientales. 


\section{AGRADECIMIENTOS}

Al licenciado Ramón Eduardo Álvarez Torres, por su apoyo y asesoramiento metodológico durante todo el proceso de la investigación y a la Dirección de Investigación Científica y Posgrado por impulsar el desarrollo de la ciencia en los estudiantes de pregrado a través de la beca que me fue otorgada.

\section{BIBLIOGRAFÍA}

Baucum, D. y Craig, G. (2009). Desarrollo psicológico. México: Pearson Educación. Baptista, P.; Fernández, C. y Hernández, R. (2007). Metodología de la investigación. México: McGraw-Hill Interamericana.

Bellido, A. y Villegas, E. (1992). Influencia de la familia en el desarrollo de pautas inadecuadas de conducta. Alternativas, Cuadernos de Trabajo Social, 1, 123-133.

Bronfenbrenner, U. (1987). La ecología del desarrollo humano. Barcelona: Paidós Ibérica.

Cadalso, R.; Cruz, R.; Fernández, O. y Noroño, N. (2002). Influencia del medio familiar en niños con conductas disruptivas. Revista Cubana de Pediatría, 74, 138-144.

Calvo, A. (2011). Conductas disruptivas y gestión eficaz del aula. Departamento de Psicología Evolutiva y de la Educación, Universidad de Murcia, España. Recuperado de: http:// www.ceptorrelavega.educantabria.es

Cava, M. (1998). La potenciación de la autoestima. Tesis doctoral. España: Universidad de Valencia.

Clemente, R., Górriz, A., Regal, A. y Villanueva, L. (2003). Interacción social entre iguales: comprensión de norma socioconvencionales y morales. España. Universitat Jaume I.

Díaz, S.; Frías, M. y López, A. (2003). Predictores de la conducta antisocial juvenil: un modelo ecológico. Estudios de Psicología, 8(1), 15-24.

Espinoza, M.; Ochaita, E. y Ortega, I. (2003). Manual formativo sobre promoción de la no violencia entre niños, niñas y adolescentes. España: Instituto Universitario de Necesidades y Derechos de la Infancia y la Adolescencia.

Estévez, E.; Jiménez, T. y Martínez, B. (2003). Influencia del funcionamiento familiar en la conducta disruptiva en adolescentes. Encuentros en Psicología Social, 1, 64-67. 
Estévez, E.; Moreno, D.; Murgui, S. y Musito, G. (2009). Relación entre el clima familiar y escolar: el rol de la empatía, la actitud hacia la autoridad y la conducta violenta en la adolescencia. International Journal of Psychology and Psychological Therapy, 9,123-136.

Farkas, C.; Freeden, P.: Grothusen, S. y Muñoz, M. (2006). Revisión de las habilidades sociocognitivas en la infancia temprana. SUMMA Psicológica UST, 3, 31-42.

Fernández, I. (2006). Guía para la convivencia en aula. Madrid: Wolters Kluwer Educación.

García, E. (2004). Conductas desadaptativas de los adolescentes en Navarra: El papel de la familia y la escuela. Tesis de doctorado. España: Universidad Pública de Navarra.

Gobierno de la República de Honduras, Secretaría Técnica de Planificación y Cooperación Externa. (2010). Visión de País 2010 - 2038 y Plan de Nación 2010- 2022. Tegucigalpa.

Gordillo, E. (2008). Relación entre el agrupamiento escolar por sexo y la frecuencia de conductas disruptivas en el aula en estudiantes de $2^{\circ}$ de secundaria en algunas instituciones educativas del área urbana del Callao. Tesis de licenciatura. Perú: Pontificia Universidad Católica del Perú.

Guitart, M. y Monreal, M. (2012). Consideraciones educativas de la perspectiva ecológica de Uri Bronfenbrenner. Contextos Educativos, 15, 79-92.

Ison, M. y Morelato, G. (2002). Contexto familiar y desarrollo de habilidades sociocognitivas para la resolución de problemas interpersonales en niños. Psykhe, 11, 149-157.

Ison, M. (2004). Características familiares y habilidades sociocognitivas en niños con conductas disruptivas. Revista Latinoamericana de Psicología, 36, 257-268.

Ison, M. y Morelato, G. (2008). Habilidades sociocognitivas en niños con conductas disruptivas y víctimas de maltrato. Universitas Psychologica, 7, 357-367.

Jiménez, M.; Lledó, A. y Sabroso, A. (2011). Problemas familiares generadores de conductas disruptivas en alumnos. International Journal of Developmental and Educational Psychology, 2, 423-432.

Luca, C.; Rodríguez, R. y Sureda, I. (2004). Programa de habilidades sociales en la enseñanza secundaria obligatoria. España: Ediciones Aljibe.

Moreno, J. y Torrejo, J. (2003). La disrupción: revisar y mejorar las estrategias de gestión del aula: interacción verbal y no verbal, discurso del profesor, estilo motivacional y reacción inmediata a la disrupción. Recuperado de: es.scribd.com Organización de las Naciones Unidas para la Educación, la Ciencia y la Cultura. (2005). Educación para todos: el imperativo de calidad. Informe de seguimiento de la EPT en el mundo. Francia. 
Sánchez, X. y Villarroel, G. (2002). Relación familia y escuela: un estudio comparativo en la ruralidad. Estudios Pedagógicos, 28, 123-141.

Sattler, J. (2010). Evaluación infantil, fundamentos cognitivos. México: Manual Moderno.

Woolfolk, A. (2010). Psicología educativa. México: Pearson Educación. 